\title{
Aspectos psicossociais no câncer pediátrico: estudo sobre literatura brasileira publicada entre 2000 e 2009
}

Psycho-social aspects of pediatric cancer: a study of the Brazilian literature published between 2000 and 2009

Aspectos psicosociales del cáncer pediátrico: estudio de la literatura brasileña publicada entre 2000 y 2009

\author{
Marina Kohlsdorf*
}

\begin{abstract}
Resumo
O câncer pediátrico representa um contexto aversivo para pacientes, familiares e profissionais de saúde. Este estudo é uma revisão da literatura brasileira publicada entre 2000 e 2009, relativa a aspectos psicossociais envolvidos no episódio de câncer pediátrico. Foi realizada busca bibliográfica nas bases de dados Scielo, Lilacs, Bireme/BVS, Pubmed/Medline, Pepsic e Psycinfo, além de busca não sistemática. Os resultados indicaram aumento dos artigos publicados nos últimos anos, predominância de relatos de pesquisa caracterizados por metodologia transversal, delineamento descritivo/exploratório, uso de análise qualitativa e técnicas de entrevista. As amostras mais pesquisadas incluíram pacientes e seus cuidadores, enquanto profissionais e irmãos de pacientes foram pouco focalizados. $\mathrm{O}$ tratamento do câncer pediátrico representa custos emocionais e instrumentais elevados para pacientes, familiares e profissionais de saúde. Destaca-se a importância de mais estudos em caráter longitudinal e que focalizem temas pouco investigados, a fim de promover intervençóes psicossociais eficientes.
\end{abstract}

Palavras-chave: câncer infantil; aspectos psicossociais; revisão de literatura.

\begin{abstract}
Pediatric cancer represents an adverse context for patients, relatives and health professionals. This paper is a review of the literature in Brazil, published between 2000 and 2009, related to psychosocial aspects involved in pediatric cancer episodes. A bibliographic review was carried out in databases Scielo, Lilacs, Bireme/BVS, Pubmed/Medline, Pepsic and Psycinfo, besides non-systematic search. The results indicated an increase in the number of published papers in the past few years, a predominance of research reports characterized by crosswise methodology, a descriptive/
\end{abstract}

Mestre em Processos de Desenvolvimento Humano e Saúde pela Universidade de Brasília (UnB).E-mail: marinak@unb.br. 
exploratory design, and the use of qualitative analysis and interview techniques. The most researched samples included patients and their caregivers, while professionals and patients' siblings were less focused on. Pediatric cancer treatment represents high emotional and instrumental costs for patients, relatives and health professionals. Emphasis is given to the relevance of further studies of a longitudinal nature taking into account poorly investigated themes, in order to promote more efficient psychosocial interventions.

Key-words: childhood cancer; psycho-social aspects; review of the literature.

\section{Resumen}

El cáncer infantil representa un contexto de aversión para los pacientes, sus familias y los profesionales de la salud. Este estudio es una revisión de la literatura brasileña, publicada entre 2000 y 2009, sobre los aspectos psicosociales involucrados en los episodios de cáncer pediátrico. Se realizó una búsqueda bibliográfica en las bases de datos Scielo, Lilacs, Bireme/BVS, Pubmed/Medline, Pepsic e Psycinfo, además de una búsqueda no sistemática. Los resultados mostraron un aumento del número de artículos publicados en los últimos años, con una prevalencia de los informes de investigación caracterizados por la metodología transversal, diseño descriptivo/exploratorio, uso del análisis cualitativo y técnicas de entrevista. Las muestras más investigadas incluyen pacientes y sus cuidadores, mientras que los profesionales y los hermanos de los pacientes son vagamente analizados. El tratamiento de cáncer pediátrico implica grandes costes emocionales e instrumentales para los pacientes, sus familias y profesionales de la salud. Se destaca la importancia de proseguir la investigación con estudios longitudinales y centrados en temas poco investigados, a fin de promover la eficacia de las intervenciones psicosociales.

Palabras clave: cáncer infantil; aspectos psicosociales; revisión de la literatura.

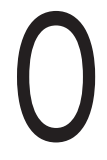

câncer pediátrico representa um grupo de doenças caracterizadas por alteraçõos na divisão e multiplicação celular, com ocorrência de metade dos casos antes dos 5 anos de idade, $25 \%$ entre 5 e 10 anos de idade e 25\% na adolescência (Inca, 2008). Segundo Gottlieb e Pinkel (1991), os principais tipos de câncer pediátrico incluem: (a) leucemias (câncer nas células da medula óssea, sendo $43 \%$ dos casos, juntamente com linfomas); (b) tumores 
do sistema nervoso central, 19\% dos casos; (c) linfomas (neoplasia no sistema linfático); (d) neuroblastomas (tumor do sistema nervoso simpático, 8\% dos casos); (e) tumor de Wilms (neoplasia renal com incidência de $6 \%$ dos casos); (f) retinoblastomas (tumor nos olhos, 3\% dos casos); (g) sarcomas (neoplasias em músculos e tendões, 7\% de casos); e (h) osteossarcomas (neoplasias nos ossos, $5 \%$ dos casos).

Costa Junior (1999; 2001a) destaca que o câncer pediátrico constitui situação de risco ao desenvolvimento, considerando as seguintes características: longo período de acompanhamento médico e internação hospitalar periódica; quimioterapia e radioterapia, com efeitos colaterais físicos e psicológicos; e exposição repetida a procedimentos médicos invasivos. Segundo o autor, pacientes e familiares precisam lidar com incertezas, riscos de recidiva da doença, alterações na rotina familiar, social, educacional e profissional, restrições físicas, experiências de ansiedade, dúvidas, perdas e mudanças na dinâmica familiar, o que justifica a atenção de profissionais de saúde, sobretudo da área de Psicologia.

\section{A Psico-oncologia Pediátrica}

Surgida na década de 50 do século XX, a Psico-oncologia caracterizase como subárea da Psicologia da saúde, de interface entre Psicologia e Oncologia, que usa conhecimentos educacionais, profissionais e científicometodológicos para identificar o papel de aspectos psicossociais na etiologia do câncer, investigar fatores psicossociais relativos à prevenção e à reabilitação, e sistematizar conhecimentos que subsidiem a assistência integral a pacientes e familiares e a qualificação profissional (Carvalho, 2002; Gimenes, 1994).

Em relação à subárea da Psico-oncologia relativa à população pediátrica, destaca-se que a área se desenvolveu nos últimos 40 anos, a partir de estudos sobre o processo de luto dos pais e reações psicológicas à morte da criança (Costa Junior, 1999; Patenaude \& Kupst, 2005). Até a década de 1960, o câncer pediátrico era considerado uma doença de evolução fatal, mas avanços científicos e tecnológicos aumentaram a eficiência dos recursos terapêuticos disponíveis, promovendo importante aumento nos índices de sobrevida ao longo das últimas décadas. Consequentemente os estudos gradualmente passaram a focalizar comportamentos e reaçôes psicológicas de pacientes, familiares e membros da equipe de saúde frente ao diagnóstico, tratamento e sobrevivência (Costa Junior, 1999; Patenaude \& Kupst, 2005).

Em investigação sobre trabalhos apresentados nos congressos brasileiros de Psico-oncologia de 1996 e 1998, Costa Junior (2001b) ressaltou que 
o atendimento psicológico a crianças com câncer foi o tema mais pesquisado (17,8\%). Kohlsdorf (2008), em análise de 129 dissertaçôes e teses produzidas entre 1993 e 2007, destacou a necessidade de ampliação dos estudos sobre aspectos psicossociais: 66 trabalhos focalizaram procedimentos médicos, pesquisas genéticas, sequelas e manifestações diagnósticas, enquanto 16 investigaram experiências de profissionais e práticas educativas. Enfrentamento, adesão, luto e autocuidados foram abordados em oito estudos, e nove trabalhos estudaram a percepção do sobrevivente. Temas menos focalizados incluíram percepção de dor, processos de comunicação, procedimentos médicos invasivos, transplante de medula óssea, reinserção escolar, satisfação com os serviços, resiliência materna, cuidados paliativos e experiências de irmãos de pacientes.

Segundo estudos de Moreira e Valle (1999), 61 trabalhos sobre aspectos psicossociais do câncer na infância foram publicados no Brasil entre 1980 e 1997, abordando temas relativos às situações vividas pela criança, seus familiares e a equipe de saúde ao longo do diagnóstico e tratamento. As autoras relatam que a compreensão da doença, além de sua esfera biológica (também em âmbito psicológico e social), torna necessário o atendimento multidisciplinar, a preparação dos profissionais $\mathrm{e} a$ atenção às reações do paciente frente ao diagnóstico e tratamento. Em revisão da literatura nacional, considerando o período entre 1998 e 2004, Silva, Teles e Valle (2005) enfatizaram elementos importantes sobre a produção de pesquisa em Psico-oncologia Pediátrica no Brasil. As autoras analisaram 56 trabalhos e constataram a predominância de estudos empíricos (40) em comparação a relatos teóricos (16), além da distribuição assistemática de trabalhos por ano. Os artigos estavam distribuídos em dez revistas científicas e havia predominância de metodologias relacionadas a abordagens qualitativas. Os temas abordados concentravam-se em (a) experiências de profissionais da enfermagem e medicina, (b) experiência de crianças com câncer, (c) impacto do tratamento para a família e (d) publicações informativas destinadas à criança, família e escola.

Destaca-se, portanto, a importância de estudos sobre revisão de literatura, em Psico-oncologia Pediátrica, que indiquem os principais temas, metodologias e resultados focalizados. Constitui objetivo deste trabalho analisar publicações brasileiras entre 2000 e 2009 que focalizaram aspectos psicossociais envolvidos no episódio de câncer pediátrico. Serão detalhados elementos superficialmente abordados em revisões tradicionais de literatura, tais como os temas focalizados, 
amostra, delineamentos metodológicos e tipos de análise, para ilustrar de modo sistemático as principais características da literatura recente.

\section{Método}

\section{Procedimento para coleta de dados}

Foi realizado levantamento bibliográfico a partir da busca eletrônica de artigos disponibilizados nas principais bases de dados: Scielo (Scientific Eletronic Library On-line), Lilacs (Literatura Latino-americana de Ciências da Saúde), Bireme/BVS (Biblioteca Virtual em Saúde), Pubmed/ Medline, Pepsic e Psycinfo, além de busca não sistemática em revistas nacionais indexadas no Portal Capes (Coordenação e Aperfeiçoamento de Pessoal de Nível Superior). As palavras-chave incluíram combinações entre câncer, leucemia, tumor, Oncologia e infantil, juvenil, infância e pediátrico. Os critérios para inclusão de dados foram: (a) artigos científicos publicados em revistas brasileiras entre 2000 e 2009; (b) modalidade de produção (artigos de pesquisa, relatos de experiência profissional, estudos de caso e teóricos, revisões de literatura); e (c) temática sobre aspectos psicossociais no câncer pediátrico.

\section{Análise dos dados}

Os artigos foram quantitativamente agrupados segundo ano e revista de publicação, tema principal, tipo de artigo (estudo teórico/revisão de literatura, relato de experiência profissional/estudo de caso e relato de pesquisa), tipo de coleta de dados (transversal, longitudinal ou acompanhamento), delineamento metodológico (descritivo/exploratório, semiexperimental ou experimental), tipo de análise de dados (qualitativa, quantitativa ou mista), instrumentos e técnicas utilizados e amostra focalizada (pacientes, profissionais de saúde e, ou, familiares). Além disso, foi realizada uma análise qualitativa sobre os principais resultados indicados pelos estudos, com base na população à qual os artigos se referiam: pacientes, familiares e profissionais de saúde.

\section{Resultados}

Descrição quantitativa dos artigos analisados

Foram localizados 66 artigos publicados em revistas brasileiras, sendo 13 artigos teóricos ou revisóes de literatura, 11 estudos de caso ou relato de 
experiência profissional e 42 relatos de pesquisa. A figura 1 indica a distribuição dos artigos por ano de publicação:

Figura 1 - Artigos por ano de publicação.

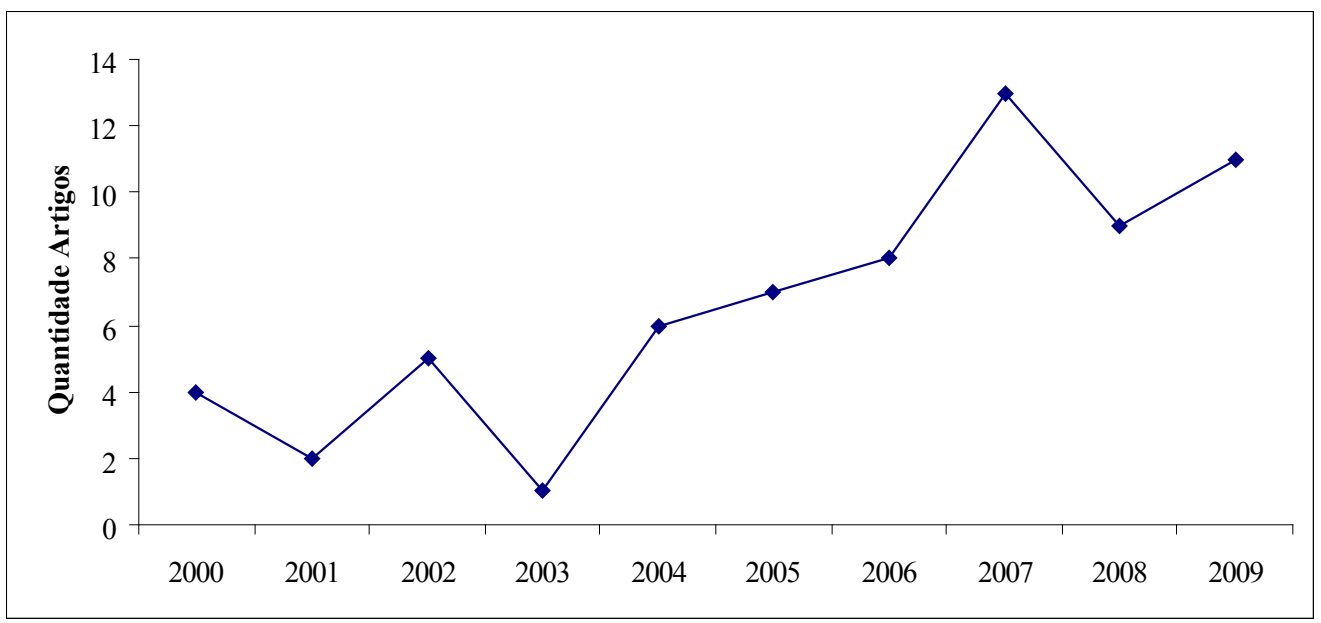

A figura 1 mostra um gradual aumento no número de publicações, especialmente a partir de 2004. As principais revistas que ofereceram artigos sobre aspectos psicossociais envolvidos no câncer pediátrico foram: Pediatria Moderna ( $\mathrm{n}=9$ ), Revista Latino-Americana de Enfermagem ( $\mathrm{n}=8)$, Psicologia em Estudo e Revista Brasileira de Cancerologia ( $\mathrm{n}=4$ cada), Estudos de Psicologia, Psicologia: Ciência e Profissão, Revista Brasileira de Enfermagem, Revista da Sociedade Brasileira de Psicologia Hospitalar (SBPH), Revista de Enfermagem da Universidade Estadual do Rio de Janeiro (UERJ) e Revista Gaúcha de Enfermagem ( $\mathrm{n}=3$ cada), Acta Oncológica, Arquivos de Ciências da Saúde, Revista da Escola Anna Nery de Enfermagem e Revista Psicologia Hospitalar ( $\mathrm{n}=2 \mathrm{cada}$ ). Outras 16 revistas publicaram um artigo cada uma. $\mathrm{O}$ quadro 1 apresenta artigos teóricos e revisôes de literatura, indicando autoria, ano de publicação, tema principal e tipo de análise:

Quadro 1 - Comparativo dos estudos e revisões de literatura no período 2000-2009

\begin{tabular}{|c|c|c|}
\hline Autor & Tema & Análise \\
\hline Araújo (2006) & $\begin{array}{l}\text { Descrever trabalhos de pesquisa do } \\
\text { Labsaudes }\end{array}$ & Qualitativa \\
\hline Costa Junior, Coutinho e Kanitz (2000) & $\begin{array}{l}\text { Analisar teoricamente enfrentamento } \\
\text { em pacientes }\end{array}$ & Qualitativa \\
\hline
\end{tabular}




\begin{tabular}{lll}
\hline Kohlsdorf e Costa Junior (2008) & $\begin{array}{l}\text { Analisar literatura sobre } \\
\text { enfrentamento }\end{array}$ & Qualitativa \\
\hline Melo e Valle (2004) & $\begin{array}{l}\text { Analisar literatura sobre morte e } \\
\text { cuidados paliativos }\end{array}$ & Qualitativa \\
\hline Menezes et alii (2007) & $\begin{array}{l}\text { Analisar literatura sobre organização } \\
\text { familiar }\end{array}$ & Qualitativa \\
\hline Menossi, Lima e Corrêa (2008) & $\begin{array}{l}\text { Analisar literatura sobre } \\
\text { interdisciplinaridade }\end{array}$ & Qualitativa \\
\hline $\begin{array}{l}\text { Nascimento, Rocha, Hayes e } \\
\text { Lima (2005) }\end{array}$ & $\begin{array}{l}\text { Analisar literatura sobre impacto } \\
\text { familiar }\end{array}$ & Qualitativa \\
\hline $\begin{array}{l}\text { Pedro, Galvão, Rocha e } \\
\text { Nascimento (2008) }\end{array}$ & $\begin{array}{l}\text { Analisar literatura sobre suporte } \\
\text { social à família }\end{array}$ & Qualitativa \\
\hline Remedi, Mello, Menossi e Lima (2009) & $\begin{array}{l}\text { Analisar literatura sobre cuidados } \\
\text { paliativos }\end{array}$ & Qualitativa \\
\hline Silva, Teles e Valle (2005) & $\begin{array}{l}\text { Analisar literatura sobre aspectos } \\
\text { psicossociais }\end{array}$ & Mista \\
\hline Souza e Erdmann (2003) & $\begin{array}{l}\text { Analisar as políticas de } \\
\text { atendimento à criança }\end{array}$ & Qualitativa \\
\hline Teles e Valle (2009) & $\begin{array}{l}\text { Analisar literatura sobre } \\
\text { sobrevivência }\end{array}$ & $\begin{array}{l}\text { Apresentar livro infantil como } \\
\text { humanização }\end{array}$ \\
\hline Veras e Moreira (2009) & Qualitativa \\
\hline
\end{tabular}

O quadro 1 indica concentração de artigos publicados em 2008 e 2009. Temas como o enfrentamento entre cuidadores e pacientes, impacto do tratamento para a família e situações extremas de sobrevivência e morte/ cuidados paliativos parecem ter sido assuntos norteadores da produção teórica brasileira durante o período analisado. Destaca-se ainda a predominância da análise qualitativa de dados em detrimento de metodologias mistas.

O quadro 2 apresenta os trabalhos descritos como estudos de caso e relatos de experiência profissional, indicando autoria, ano de publicação, tema principal, tipo de análise de dados, instrumentos e, ou, técnicas utilizados e participantes:

Quadro 2 - Comparativo dos estudos de caso e relatos de experiência profissional

\begin{tabular}{lllll}
\hline \multicolumn{1}{c}{ Autor } & \multicolumn{1}{c}{ Tema } & Análise & $\begin{array}{c}\text { Instrumentos e } \\
\text { Técnicas }\end{array}$ & Participantes \\
\hline $\begin{array}{l}\text { Bolze e Castoldi } \\
(2005)^{*}\end{array}$ & $\begin{array}{l}\text { Intervenção e } \\
\text { óbito }\end{array}$ & Qualitativa & Acompanhamento & $\begin{array}{l}\text { Pais e quatro } \\
\text { filhos }\end{array}$ \\
\hline $\begin{array}{l}\text { Campos et alii } \\
(2007)^{* *}\end{array}$ & $\begin{array}{l}\text { Intervenção e } \\
\text { transplante }\end{array}$ & Qualitativa & Grupo focal & Mães \\
\hline
\end{tabular}




\begin{tabular}{|c|c|c|c|c|}
\hline Carlesso (2008)* & $\begin{array}{l}\text { Intervenção e } \\
\text { óbito }\end{array}$ & Qualitativa & Acompanhamento & Mãe e criança \\
\hline Costa $(2002)^{* *}$ & Atividades lúdicas & Qualitativa & NSA & NSA \\
\hline $\begin{array}{l}\text { Costa Junior et alii } \\
(2006)^{* *}\end{array}$ & $\begin{array}{l}\text { Recreação plane- } \\
\text { jada }\end{array}$ & Mista & NSA & NSA \\
\hline $\begin{array}{l}\text { Espíndula e Valle } \\
(2002)^{*}\end{array}$ & $\begin{array}{l}\text { Vivência frente à } \\
\text { recidiva }\end{array}$ & Qualitativa & Entrevista & Uma mãe \\
\hline $\begin{array}{l}\text { Herman e Miyazaki } \\
(2007)^{*}\end{array}$ & $\begin{array}{l}\text { Programa psico- } \\
\text { educacional }\end{array}$ & Mista & $\begin{array}{l}\text { Inventário estilo pa- } \\
\text { rental, entrevista, in- } \\
\text { ventário de compor- } \\
\text { tamentos na infância } \\
\text { e adolescência }\end{array}$ & Uma mãe \\
\hline $\begin{array}{l}\text { Morais e Valle } \\
(2001)^{*}\end{array}$ & $\begin{array}{l}\text { Terapia ocupacio- } \\
\text { nal }\end{array}$ & Qualitativa & Observação & Uma criança \\
\hline $\begin{array}{l}\text { Pedrosa et alii } \\
(2007)^{* *}\end{array}$ & Atividades lúdicas & Mista & Questionário & 60 pacientes \\
\hline $\begin{array}{l}\text { Rodrigues, Culau e } \\
\text { Nunes }(2007)^{* *}\end{array}$ & $\begin{array}{l}\text { Percepções sobre } \\
\text { cuidado }\end{array}$ & Qualitativa & Diários de campo & $\begin{array}{l}12 \text { estudantes } \\
\text { de Enfermagem }\end{array}$ \\
\hline $\begin{array}{l}\text { Silva e Valle } \\
(2005)^{* *}\end{array}$ & \multicolumn{2}{|c|}{ Reinserção escolar Qualitativa } & Entrevista & Oito crianças \\
\hline
\end{tabular}

Notas: * estudos de caso clínico; ${ }^{* *}$ relatos de experiência profissional; NSA = não se aplica.

As publicações descritas como estudos de caso ou relato de experiência profissional apresentaram aumento de publicações ao longo do período ( $\mathrm{n}=1$ em 2001 e n = 5 em 2007 e 2008). Ressalta-se o predomínio de estudos descrevendo atuação profissional junto aos cuidadores e pacientes $(n=6)$, além de situações especificamente críticas como transplante de medula óssea e óbito do paciente. Dos 11 trabalhos analisados com essa temática, oito focalizaram a metodologia qualitativa e três estudos indicaram análise mista dos dados.

Os quadros 3 e 4 apresentam os trabalhos descritos como relatos de pesquisa, indicando autoria, ano de publicação, tema, tipo de coleta de dados, delineamento metodológico, tipo de análise, instrumentos e, ou, técnicas utilizados e participantes: 
Quadro 3 - Comparativo de relatos de pesquisa publicados entre 2000 e 2006

\begin{tabular}{|c|c|c|c|c|c|}
\hline Autor & Tema & Delineamento & Análise & $\begin{array}{l}\text { Instrumentos } \\
\text { e técnicas }\end{array}$ & Participantes \\
\hline $\begin{array}{l}\text { Camacho } \\
(2006)^{* *}\end{array}$ & $\begin{array}{l}\text { Impacto do } \\
\text { câncer }\end{array}$ & $\mathrm{DE}$ & Qualitativa & $\begin{array}{l}\text { Diários de } \\
\text { campo }\end{array}$ & $\begin{array}{l}83 \text { pacientes, } 39 \\
\text { familiares, } 22 \\
\text { profissionais }\end{array}$ \\
\hline $\begin{array}{l}\text { Capitão e Zam- } \\
\text { pronha }(2004)^{*}\end{array}$ & $\begin{array}{l}\text { Impacto do } \\
\text { câncer }\end{array}$ & DE & Quantitativa & $\begin{array}{l}\text { Questionário } \\
\text { Desiderativo }\end{array}$ & 8 adolescentes \\
\hline $\begin{array}{l}\text { Costa e Lima } \\
(2002)^{*}\end{array}$ & $\begin{array}{l}\text { Impacto } \\
\text { doméstico }\end{array}$ & DE & Qualitativa & Entrevistas & 15 cuidadores \\
\hline $\begin{array}{l}\text { Costa Junior e } \\
\text { Kanitz }(2000)^{* *}\end{array}$ & $\begin{array}{l}\text { Programa de } \\
\text { intervenção }\end{array}$ & Experimental & Mista & $\begin{array}{l}\text { Videogravação } \\
\text { e entrevista }\end{array}$ & 20 crianças \\
\hline $\begin{array}{l}\text { Costa Junior, } \\
\text { Coutinho e Fer- } \\
\text { reira }(2006)^{*}\end{array}$ & $\begin{array}{l}\text { Recreação } \\
\text { planejada }\end{array}$ & $\begin{array}{l}\text { Quase experi- } \\
\text { ental }\end{array}$ & Quantitativo & Observação & $\begin{array}{l}91 \text { crianças e } \\
\text { adolescentes }\end{array}$ \\
\hline $\begin{array}{l}\text { Delella e Araújo } \\
(2002)^{*}\end{array}$ & $\begin{array}{l}\text { Estresse pós- } \\
\text { traumático }\end{array}$ & $\mathrm{DE}$ & Mista & $\begin{array}{l}\text { Entrevista } \\
\text { clínica }\end{array}$ & $\begin{array}{l}10 \text { crianças e } \\
\text { seus pais }\end{array}$ \\
\hline $\begin{array}{l}\text { Fontenele et } \\
\text { alii }(2000)^{*}\end{array}$ & $\begin{array}{l}\text { Recursos } \\
\text { lúdicos }\end{array}$ & DE & Qualitativa & $\begin{array}{l}\text { Entrevistas } \\
\text { observação }\end{array}$ & $\begin{array}{l}13 \text { crianças } \\
\text { hospitalizadas }\end{array}$ \\
\hline $\begin{array}{l}\text { Jesus e Gon- } \\
\text { çalves (2006)* }\end{array}$ & $\begin{array}{l}\text { Impacto } \\
\text { cotidiano }\end{array}$ & DE & Qualitativa & Entrevista & 7 adolescentes \\
\hline $\begin{array}{l}\text { Lemos, Lima e } \\
\text { Mello }(2004)^{*}\end{array}$ & $\begin{array}{l}\text { Impacto } \\
\text { tratamento }\end{array}$ & DE & Qualitativa & Entrevistas & 11 pacientes \\
\hline $\begin{array}{l}\text { Marques } \\
(2004)^{*}\end{array}$ & $\begin{array}{l}\text { Estresse } \\
\text { infantil }\end{array}$ & $\mathrm{DE}$ & Mista & $\begin{array}{l}\text { Escala de es- } \\
\text { tresse infantil, } \\
\text { entrevista }\end{array}$ & 25 crianças \\
\hline $\begin{array}{l}\text { Motta e Enumo } \\
(2002)^{*}\end{array}$ & $\begin{array}{l}\text { Enfrentam- } \\
\text { ento entre } \\
\text { pacientes }\end{array}$ & DE & Quantitativa & $\begin{array}{l}\text { Avaliação, } \\
\text { estratégias de } \\
\text { enfrentam- } \\
\text { ento, hospital- } \\
\text { ização }\end{array}$ & 28 crianças \\
\hline $\begin{array}{l}\text { Motta e Enumo } \\
(2004 a)^{*}\end{array}$ & $\begin{array}{l}\text { Estratégias } \\
\text { de enfren- } \\
\text { tamento em } \\
\text { pacientes }\end{array}$ & DE & Quantitativa & $\begin{array}{l}\text { Avaliação, } \\
\text { estratégias de } \\
\text { enfrentam- } \\
\text { ento, hospital- } \\
\text { ização }\end{array}$ & 28 crianças \\
\hline $\begin{array}{l}\text { Motta e Enumo } \\
(2004 b)^{*}\end{array}$ & $\begin{array}{l}\text { Estratégias } \\
\text { de enfren- } \\
\text { tamento em } \\
\text { pacientes }\end{array}$ & DE & Quantitativa & $\begin{array}{l}\text { Avaliação, } \\
\text { estratégias de } \\
\text { enfrentam- } \\
\text { ento, hospital- } \\
\text { ização }\end{array}$ & 28 crianças \\
\hline $\begin{array}{l}\text { Paro, Paro } \\
\text { e Ferreira } \\
(2005)^{*}\end{array}$ & $\begin{array}{l}\text { Ações em } \\
\text { cuidado }\end{array}$ & DE & Qualitativa & Entrevista & 17 enfermeiras \\
\hline $\begin{array}{l}\text { Pedro e } \\
\text { Funghetto } \\
(2005)^{*}\end{array}$ & $\begin{array}{l}\text { Ações em } \\
\text { cuidado }\end{array}$ & $\mathrm{DE}$ & Qualitativa & Entrevista & 15 enfermeiros \\
\hline
\end{tabular}




\begin{tabular}{llllll}
\hline $\begin{array}{l}\text { Pedrosa e Valle } \\
(2000)^{*}\end{array}$ & $\begin{array}{l}\text { Impacto } \\
\text { tratamento }\end{array}$ & DE & Qualitativa & Entrevista & 11 irmãos \\
\hline $\begin{array}{l}\text { Ribeiro e Rodri- } \\
\text { gues (2005)* }\end{array}$ & $\begin{array}{l}\text { Impacto } \\
\text { tratamento }\end{array}$ & DE & Qualitativa & Entrevista & 16 adolescentes \\
\hline $\begin{array}{l}\text { Silva, Gallego e } \\
\text { Teixeira (2006)* }\end{array}$ & $\begin{array}{l}\text { Habilidades } \\
\text { intelectuais }\end{array}$ & DE & Quantitativa & $\begin{array}{l}\text { Escala de } \\
\text { Inteligência } \\
\text { Wechsler }\end{array}$ & $\begin{array}{l}\text { 8 pacientes, } 9 \\
\text { crianças sau- } \\
\text { dáveis }\end{array}$ \\
\hline $\begin{array}{l}\text { Silveira e Lima } \\
\text { (2001)* }\end{array}$ & $\begin{array}{l}\text { Casas de } \\
\text { apoio }\end{array}$ & DE & Mista & Questionário & 28 instituições \\
\hline $\begin{array}{l}\text { Steffen e Cas- } \\
\text { toldi (2006)* }\end{array}$ & $\begin{array}{l}\text { Dinâmica } \\
\text { conjugal }\end{array}$ & DE & Qualitativa & Entrevista & Três casais \\
\hline $\begin{array}{l}\text { Vasconcelos, } \\
\begin{array}{l}\text { Albuquerque e } \\
\text { Costa (2006)* }\end{array}\end{array}$ & $\begin{array}{l}\text { Terapia } \\
\text { ocupacional }\end{array}$ & DE & Qualitativa & Entrevista & $\begin{array}{l}\text { 6 crianças e } \\
\text { suas mães }\end{array}$ \\
\hline
\end{tabular}

Notas: ${ }^{*}$ estudos transversais; ${ }^{* *}$ estudos de Acompanhamento; $\mathrm{DE}=$ descritivo/exploratório.

Quadro 4 - Comparativo de relatos de pesquisa publicados entre 2007 e 2009

\begin{tabular}{|c|c|c|c|c|c|}
\hline Autor & Tema & Delineamento & Análise & $\begin{array}{l}\text { Instrumentos } \\
\text { e Técnicas }\end{array}$ & Participantes \\
\hline $\begin{array}{l}\text { Araujo e } \\
\text { Guimarães } \\
(2009)^{*}\end{array}$ & $\begin{array}{l}\text { "Palhaços- } \\
\text { doutores" e } \\
\text { enfrentamento }\end{array}$ & $\mathrm{DE}$ & Qualitativa & $\begin{array}{l}\text { Entrevistas, } \\
\text { observação }\end{array}$ & $\begin{array}{l}11 \text { pacientes, } \\
21 \text { cuidadores, } \\
2 \text { profissionais }\end{array}$ \\
\hline $\begin{array}{l}\text { Avanci et alii } \\
(2009)^{*}\end{array}$ & $\begin{array}{l}\text { Cuidados } \\
\text { paliativos }\end{array}$ & $\mathrm{DE}$ & Qualitativa & Entrevistas & 5 enfermeiros \\
\hline $\begin{array}{l}\text { Beck e Lopes } \\
(2007 a)^{*}\end{array}$ & $\begin{array}{l}\text { Impacto } \\
\text { tratamento }\end{array}$ & $\mathrm{DE}$ & $\begin{array}{l}\text { Quantita- } \\
\text { tiva }\end{array}$ & Entrevista & 50 cuidadores \\
\hline $\begin{array}{l}\text { Beck e Lopes } \\
(2007 b)^{*}\end{array}$ & $\begin{array}{l}\text { Tensão } \\
\text { cuidadores }\end{array}$ & $\mathrm{DE}$ & Mista & Entrevistas & 50 cuidadores \\
\hline $\begin{array}{l}\text { Beltrão, Vascon- } \\
\text { celos, Pontes } \\
\text { e Albuquerque } \\
(2007)^{*}\end{array}$ & $\begin{array}{l}\text { Enfrentamento } \\
\text { em cuidadores }\end{array}$ & $\mathrm{DE}$ & Qualitativa & $\begin{array}{l}\text { Entrevistas, } \\
\text { observação }\end{array}$ & 10 mães \\
\hline $\begin{array}{l}\text { Cavicchioli, } \\
\text { Menossi e Lima } \\
(2007)^{*}\end{array}$ & $\begin{array}{l}\text { Trajetória até } \\
\text { diagnóstico }\end{array}$ & $\mathrm{DE}$ & Qualitativa & Entrevistas & 10 cuidadores \\
\hline $\begin{array}{l}\text { Ferreira e Garcia } \\
(2008)^{*}\end{array}$ & Interação social & $\mathrm{DE}$ & Qualitativa & Entrevistas & $\begin{array}{l}3 \text { adolescentes } \\
\text { e } 3 \text { amigos }\end{array}$ \\
\hline $\begin{array}{l}\text { Freire, Petrilli } \\
\text { e Sonsogno } \\
(2007)^{*}\end{array}$ & Humanização & $\mathrm{DE}$ & Mista & $\begin{array}{l}\text { Entrevistas, } \\
\text { observação }\end{array}$ & 30 mães \\
\hline $\begin{array}{l}\text { Malta, Schall e } \\
\text { Modena }(2008)^{*}\end{array}$ & $\begin{array}{l}\text { Impacto } \\
\text { tratamento }\end{array}$ & $\mathrm{DE}$ & Qualitativa & Entrevistas & 16 cuidadores \\
\hline
\end{tabular}




\begin{tabular}{|c|c|c|c|c|c|}
\hline $\begin{array}{l}\text { Malta, Schall } \\
\text { e Modena } \\
(2009 a)^{*}\end{array}$ & $\begin{array}{l}\text { Percepções de } \\
\text { crianças }\end{array}$ & $\mathrm{DE}$ & Qualitativa & $\begin{array}{l}\text { Entrevistas, } \\
\text { observação }\end{array}$ & 12 crianças \\
\hline $\begin{array}{l}\text { Malta, Schall } \\
\text { e Modena } \\
(2009 b)^{*}\end{array}$ & $\begin{array}{l}\text { Comunicação } \\
\text { de diagnóstico }\end{array}$ & DE & Qualitativa & Entrevistas & 5 oncologistas \\
\hline $\begin{array}{l}\text { Marchesan et } \\
\text { alii }(2009)^{*}\end{array}$ & $\begin{array}{l}\text { Escola } \\
\text { hospitalar }\end{array}$ & $\mathrm{DE}$ & Qualitativa & Entrevistas & 28 pacientes \\
\hline $\begin{array}{l}\text { Mensório, Kohls- } \\
\text { dorf e Costa } \\
\text { Junior }(2009)^{* *}\end{array}$ & $\begin{array}{l}\text { Mudanças no } \\
\text { enfrentamento } \\
\text { em cuidadores }\end{array}$ & $\mathrm{DE}$ & Mista & $\begin{array}{l}\text { Escala Modos } \\
\text { Enfrentamento } \\
\text { Problemas, } \\
\text { Inventário } \\
\text { Beck Ansie- } \\
\text { dade }\end{array}$ & 30 cuidadores \\
\hline $\begin{array}{l}\text { Misko e Bousso } \\
(2007)^{*}\end{array}$ & $\begin{array}{l}\text { Manejo } \\
\text { doméstico }\end{array}$ & DE & Qualitativa & Entrevista & 6 mães \\
\hline $\begin{array}{l}\text { Moreira e Ân- } \\
\text { gelo }(2008)^{*}\end{array}$ & $\begin{array}{l}\text { Desafios para } \\
\text { mães }\end{array}$ & $\mathrm{DE}$ & Qualitativa & Entrevista & 7 mães \\
\hline $\begin{array}{l}\text { Mussa e Malerbi } \\
(2008)^{*}\end{array}$ & $\begin{array}{l}\text { Atividades } \\
\text { lúdicas e } \\
\text { queixas de dor }\end{array}$ & $\begin{array}{l}\text { Quase } \\
\text { experimental }\end{array}$ & Mista & $\begin{array}{l}\text { Entrevista, } \\
\text { observa- } \\
\text { ção, escala } \\
\text { análogo-visual } \\
\text { dor }\end{array}$ & $\begin{array}{l}15 \text { crianças e } \\
\text { cuidadores }\end{array}$ \\
\hline $\begin{array}{l}\text { Ortiz e Lima } \\
(2007)^{*}\end{array}$ & Sobrevivência & $\mathrm{DE}$ & Qualitativa & Entrevista & $\begin{array}{l}9 \text { mães, } \\
2 \text { pais, } 2 \text { irmãs }\end{array}$ \\
\hline $\begin{array}{l}\text { Ramalho e } \\
\text { Nogueira-Mar- } \\
\text { tins }(2007)^{*}\end{array}$ & $\begin{array}{l}\text { Realidade ocu- } \\
\text { pacional }\end{array}$ & DE & Qualitativa & Entrevista & 9 profissionais \\
\hline $\begin{array}{l}\text { Santos e Gon- } \\
\text { çalves }(2008)^{*}\end{array}$ & $\begin{array}{l}\text { Significado } \\
\text { doença }\end{array}$ & DE & Qualitativa & Entrevista & 10 mães \\
\hline $\begin{array}{l}\text { Silva et alii } \\
(2009)^{*}\end{array}$ & $\begin{array}{l}\text { Significado } \\
\text { doença }\end{array}$ & DE & Mista & Entrevista & $\begin{array}{l}\text { Familiares de } \\
54 \text { pacientes }\end{array}$ \\
\hline $\begin{array}{l}\text { Wegner e Pedro } \\
(2009)^{* *}\end{array}$ & $\begin{array}{l}\text { Impacto } \\
\text { tratamento }\end{array}$ & $\mathrm{DE}$ & Qualitativa & Grupo Focal & 9 cuidadoras \\
\hline
\end{tabular}

Nota: * estudos transversais; ${ }^{* *}$ estudos de acompanhamento; $\mathrm{DE}=$ descritivo/ exploratório.

Os quadros 3 e 4 indicam distribuição heterogênea dos trabalhos: no período inicial de sete anos (2000 a 2006), foram publicados 21 estudos, já no período final de apenas dois anos (2007-2009), foi publicada a mesma quantidade de 21 artigos. Investigaçóes sobre o impacto do tratamento para cuidadores, pacientes e, em menor grau, profissionais de saúde foram os temas mais focalizados nas publicaçôes, com poucos relatos sobre intervenções. Destaca-se a prevalência de estudos de caráter descritivo/exploratório ( $\mathrm{n}=$ 39), em detrimento de delineamentos de pesquisa experimentais ou quase experimentais $(\mathrm{n}=3)$. Análises qualitativas de dados caracterizaram-se como 
maioria $(n=26)$, mas análises quantitativas $(n=7)$ e mistas $(n=9)$ também foram usadas e entrevistas constituíram a técnica mais empregada nos estudos $(n=31)$. A figura 2 indica a população focalizada, considerando os artigos de pesquisa, relatos de experiência profissional e estudos de caso:

Figura 2 - População focalizada nos estudos.

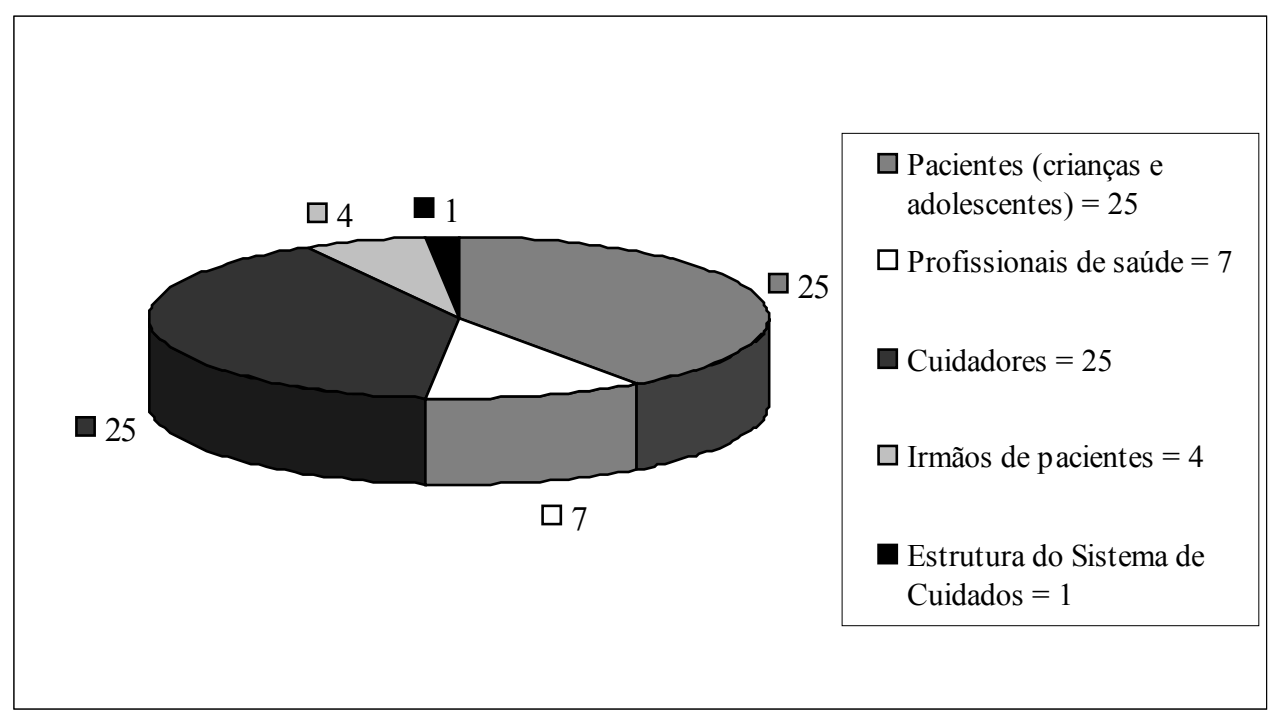

Nota: $\mathrm{n}=62$, pois oito artigos incluíram mais de um tipo de população.

A figura 2 indica concentração de estudos sobre pacientes e cuidadores pediátricos $(\mathrm{n}=25 \mathrm{em}$ cada). Profissionais e irmãos de pacientes foram incluídos em poucos estudos (sete e quatro trabalhos, respectivamente) e apenas um artigo abordou a estrutura de sistema de cuidados, descrevendo o funcionamento de casas de apoio para permanência de pacientes e cuidadores (locais que oferecem moradia para pais e pacientes provenientes de outros Estados brasileiros diferentes daquele onde se realiza o tratamento).

\section{Descrição qualitativa dos artigos analisados}

Considerando que o tratamento do câncer pediátrico envolve essencialmente uma tríade formada por pacientes, familiares e profissionais de saúde, são destacadas a seguir as principais contribuiçóes dos artigos analisados em relação aos aspectos psicossociais envolvidos na vivência de cada uma dessas amostras, durante o episódio de câncer pediátrico. 


\section{Impacto do episódio de câncer pediátrico para pacientes}

Os trabalhos destacam as exigências vivenciadas pelas crianças, especialmente relacionadas à quimioterapia e à hospitalização, contexto que inclui perdas sociais, experiências de dor física, medo e efeitos colaterais, incertezas, ansiedade e restrições no cotidiano, o que propicia níveis importantes de estresse (Jesus \& Gonçalves, 2006; Lemos, Lima \& Mello, 2004; Marques, 2004). Malta, Schall, Reis e Modena (2009) destacam a dificuldade de a criança falar sobre a doença, com narrativas baseadas na reprodução do discurso biomédico, impacto dos efeitos colaterais e dificuldade em manifestar sentimentos, sendo a brincadeira a principal forma de expressão infantil.

Motta e Enumo (2002; 2004a; 2004b) também destacam a preferência da criança pelo brincar como estratégia adaptativa. As autoras indicam que crianças nesse contexto tendem a desenvolver estratégias mais positivas para lidar com as dificuldades, tais como brincar, ler, conversar, seguir a medicação e rezar, em detrimento de estratégias negativas (como se esconder, sentir culpa, brigar e chantagear). Marchesan, Bock, Petrilli, Covic \& Kanemoto (2009) indicam a importância de promover estratégias que possam aproximar a vivência da criança em tratamento do que seria seu curso de desenvolvimento sem o episódio de câncer, por exemplo, desde a adaptação da vivência escolar.

Costa Junior et alii (2006) e ainda Costa Junior e Kanitz (2000) destacam a importância das atividades de recreação planejada em sala de espera para auxiliar as crianças a lidar melhor com os desafios do tratamento e da hospitalização. Os trabalhos desses autores indicam que tais atividades possibilitam a ampliação do repertório de comportamentos colaborativos, de interação social e de adaptação aos eventos adversos provenientes do tratamento. A intervenção de "palhaços-doutores" é indicada por Araújo e Guimarães (2009) como um elemento benéfico ao enfrentamento da hospitalização, como estratégia distrativa e de estimulação do desenvolvimento em condições adversas.

A terapia ocupacional e atividades lúdicas envolvendo a literatura infanto-juvenil têm sido apontadas como relevantes, ao auxiliar a criança a se aproximar das dificuldades e proporcionar a percepção em relação ao seu corpo, às suas respostas emocionais e às suas expectativas (Araújo, 2006; Costa, 2002; Fontenele et alii, 2000; Morais \& Valle 2001; Pedrosa, Monteiro, Lins, Pedrosa \& Melo, 2007; Vasconcelos, Albuquerque \& Costa, 2006; Veras \& Moreira, 2009). Mussa e Malerbi (2004) destacam 
que o trabalho de contadores de histórias influencia, de forma benéfica, a adaptação da criança ao tratamento, pois os pacientes passam a apresentar menos queixa de dor, maior interação social, colaboração com procedimentos, movimentação pela enfermaria e aceitação de alimentos.

Constitui foco especial de estudo a percepção do paciente adolescente, tema abordado em apenas quatro estudos. Para o adolescente, o contexto de tratamento é caracterizado pela aversividade de efeitos colaterais e procedimentos médicos, incerteza sobre a possibilidade de cura e sequelas, desvalorização pessoal, sensação de vulnerabilidade, ameaça e fragilidade, prejuízos em relações interpessoais, transformação da rotina, preocupação com familiares, expectativas de cura e retorno às atividades cotidianas, medo de falecimento, dificuldades para verbalizar sentimentos e prejuízos ao desenvolvimento (Capitão \& Zampronha, 2004; Ferreira \& Garcia, 2008; Jesus \& Gonçalves, 2006; Ribeiro \& Rodrigues, 2005).

\section{Impacto do episódio de câncer pediátrico para familiares}

Nesse contexto, os estudos focalizam prioritariamente a vivência dos cuidadores, em especial mães (geralmente o cuidador primário). O contexto aversivo se inicia antes mesmo da confirmação diagnóstica, pois pacientes e pais precisam enfrentar um período muitas vezes longo e marcado por incertezas e expectativas, desde o reconhecimento de sinais e sintomas, hipóteses diagnósticas, dificuldades para realizar exames e encaminhar-se ao serviço especializado (Cavicchioli, Menossi \& Lima, 2007; Malta, Schall \& Modena, 2008).

Durante o tratamento, os cuidadores acumulam as funções educativas parentais e a administração de suas próprias condições emocionais. $\mathrm{O}$ impacto do diagnóstico e tratamento inclui mudanças na dinâmica familiar, alterações na relação conjugal, prejuízos em atividades sociais, profissionais e de autocuidado, administração de intercorrências e emergências, sobrecarga de cuidados, alterações em práticas parentais, manejo da relação com os filhos saudáveis, vivências de medo, tensão, resignação, ansiedade ou incerteza e ainda dificuldades financeiras (Beck \& Lopes, 2007a; 2007b; Costa \& Lima, 2002; Herman \& Miyazaki, 2007; Misko \& Bousso, 2007; Moreira \& Ângelo, 2008; Santos \& Gonçalves, 2008; Silva, Andrade, Barbosa, Hoffman \& Macedo, 2009; Steffen \& Castoldi, 2006).

Diante dos desafios impostos pelo tratamento, os cuidadores usam estratégias que incluem busca por suporte social, busca por informações sobre a doença, aumento de práticas religiosas e seguimento das recomendações 
de cuidados (Beck \& Lopes, 2007a; 2007b; Beltrão, Vasconcelos, Pontes \& Albuquerque, 2007; Freire, Petrilli \& Sonsogno, 2007; Malta et alii, 2008; Mensório, Kohlsdorf \& Costa Junior, 2009; Pedro, Galvão, Rocha \& Nascimento, 2008; Santos \& Gonçalves, 2008). Silveira e Lima (2001) destacam a importância das casas de apoio como suporte instrumental e ressaltam que a qualidade desse ambiente físico é primordial.

Os estudos indicam intervençôes relevantes para diminuir os custos emocionais associados ao tratamento e facilitar a adaptação dos cuidadores ao contexto aversivo. Intervençôes em grupos focais têm sido apontadas como benéficas para mães de pacientes, pois essas modalidades de intervenção propiciam o fornecimento de suporte social proveniente de outras cuidadoras em situação semelhante, promovendo uma atuação mais ativa das mães no tratamento (Campos, Rodrigues, Machado \& Alvarez, 2007; Wegner \& Pedro, 2009). A terapia ocupacional e a interação com "palhaços-doutores" também são estratégias distrativas para enfrentamento de condições adversas (Araújo \& Guimarães, 2009; Vasconcelos et alii, 2006). Herman e Miyazaki (2007) destacam que programas educativos são úteis para proporcionar o ajuste de práticas parentais, promovendo a adaptação e desenvolvimento de pais e pacientes.

Beltrão et alii (2007) e ainda Pedro et alii (2008) destacam que a fase diagnóstica é o período mais difícil, mas, mesmo após a conclusão do tratamento, a experiência do câncer é revestida por intenso aspecto negativo. A reinserção escolar, a possibilidade de recidiva, a readaptação social do paciente e as possíveis sequelas do tratamento são fatores estressores para cuidadores (Beck \& Lopes, 2007a; 2007b; Dellela \& Araújo, 2002; Espíndula \& Valle, 2002; Ortiz \& Lima, 2007; Silva \& Valle, 2005). Por outro lado, Delella e Araújo (2002) e ainda Teles e Valle (2009) indicam que pais de sobreviventes podem apresentar boa adaptação ao contexto, com pouco prejuízo ao funcionamento psicossocial. Cabe destacar ainda que cuidados paliativos e morte de pacientes são contextos extremamente aversivos para cuidadores. Carlesso (2008) descreve a angústia e o sofrimento extremos diante da morte do filho, e Bolze e Castoldi (2005) destacam a importância da atuação do psicólogo junto aos pais quando iniciados os cuidados paliativos.

Estudos com irmãos de pacientes em tratamento também são de grande importância, embora a literatura seja bastante escassa em relação a esse tema. Pedrosa e Valle (2000) destacam que o irmão do paciente percebe as transformações domésticas e emocionais no contexto familiar, sendo diretamente influenciado pelo tratamento. As autoras destacam ainda que 
os irmãos sadios podem experimentar sentimentos de angústia, medo, ansiedade, transformações em relacionamentos interpessoais e dificuldades de ajustamento.

\section{Impacto do episódio de câncer pediátrico para profissionais}

A vivência dos profissionais de saúde em episódios de câncer pediátrico tem sido pouco investigada. Remedi, Mello, Menossi e Lima (2009) destacam a escassez de estudos na área, com ausência de programas específicos para um contexto que demanda esforços adaptativos por parte dos profissionais. $\mathrm{O}$ caráter prolongado do tratamento exige que pacientes e familiares permaneçam em contato com a equipe de saúde, propiciando a formação de vínculos importantes, o estabelecimento de relações afetivas e, de modo especialmente relevante, o fornecimento de suporte social ao paciente e familiares, em um contexto potencialmente estressante e permeado por perdas (Pedro \& Funghetto, 2005; Rodrigues, Culau \& Nunes, 2007; Silva et alii, 2009).

De modo particular, o contexto de cuidados paliativos se apresenta como crítico. Avanci, Carolindo, Góes e Netto (2009), Melo e Valle (2004) e Paro et alii (2005) apontam que muitas vezes o profissional tem limitaçôes para lidar com cuidados paliativos e morte de crianças. Os autores ressaltam que cuidar de um paciente sob cuidados paliativos é um processo de mobilização intensa, em que os profissionais experimentam emoções ambivalentes em um contexto que deve estar voltado para a promoção de conforto, alívio de dor e atendimento às necessidades da família. $\mathrm{O}$ fornecimento de suporte social, a humanização e a interdisciplinaridade são elementos fundamentais para diminuir os custos emocionais vivenciados pelos profissionais (Malta, Schall \& Modena, 2009; Menossi, Lima \& Corrêa, 2008; Paro et alii, 2005; Pedro \& Funghetto, 2005).

Além dos custos emocionais envolvidos no cuidado a pacientes em tratamento e suas famílias, Paro et alii (2005) e ainda Ramalho e Nogueira-Martins (2007) destacam como fontes importantes de estresse as dificuldades da organização do trabalho do enfermeiro. Entre os principais desafios, os autores destacam a falta de reconhecimento do trabalho, problemas na rotina, falhas na coordenação do grupo, falta de recursos para auxílio ao profissional e estrutura administrativa falha. Os autores destacam a importância de intervençôes organizacionais que possam capacitar o profissional a desenvolver seu trabalho de forma satisfatória, prevenindo a instalação do estresse, promovendo recursos de 
apoio e segurança, possibilitando a implantação de cuidados humanizados e interdisciplinares e permitindo ao profissional a revisão periódica de seus conhecimentos.

Especificamente em relação aos médicos oncologistas pediátricos, Melo e Valle (2004) e Malta, Schall e Modena (2009) indicam que o momento de comunicação de diagnóstico é desafiador. As dificuldades incluem a forma de comunicar o diagnóstico, a inclusão do paciente na transmissão da informação, as dificuldades na organização e estrutura do serviço (preparação pessoal e do lugar físico), o acolhimento das respostas emocionais de pacientes e cuidadores, e ainda a escassez de ações eficientes de humanização no cuidado.

\section{Discussão}

Os resultados deste estudo ressaltam o aumento gradual de trabalhos sobre aspectos psicossociais do câncer pediátrico, corroborando os trabalhos de Moreira e Valle (1999) e Silva et alii (2005). Destaca-se a predominância de artigos de pesquisa $(n=42)$ em relação a outros tipos de trabalho como relatos de experiência profissional, estudos teóricos/revisões de literatura e estudos de caso ( $\mathrm{n}=23)$, como também indicado por Silva et alii (2005).

Os temas abordados pelos estudos focalizaram principalmente a experiência de pacientes e cuidadores. Destaca-se a necessidade de estudos que investiguem temas essenciais e pouco avaliados, como a experiência de profissionais, adesão ao tratamento, cuidados paliativos, experiência do adolescente, impacto do tratamento para irmãos de pacientes, percepção de sobreviventes, entre outros. Cabe ressaltar também a escassez de estudos com delineamento experimental ou quase experimental que possam contribuir de forma relevante para a compreensão sobre quais elementos estão funcionalmente envolvidos no tratamento do câncer pediátrico e intervenções que possam diminuir custos comportamentais.

Ressalta-se que nenhum estudo nesta amostra foi caracterizado como longitudinal. Conforme apontado por Delella e Araújo (2002), estudos em caráter longitudinal são fundamentais neste contexto, considerando que o episódio de câncer pediátrico envolve um período prolongado desde o diagnóstico até a finalização do tratamento. Destaca-se a predominância de trabalhos com metodologia qualitativa e técnicas de entrevista, resultado que aponta a necessidade de diversificação dos tipos de análise e instrumentos ou técnicas utilizadas, além da validação de mais instrumentos psicométricos para a população brasileira. 
O episódio de câncer pediátrico é um contexto potencialmente estressante e aversivo para pacientes, familiares e profissionais de saúde. Torna-se imprescindível que pesquisadores brasileiros ampliem os estudos na área e abordem fatores psicossociais neste contexto. Conforme indicado por Silveira e Lima (2001) e também Souza e Erdmann (2003), a compreensão da vivência de pacientes, familiares e profissionais deve envolver aspectos globais (relativos à organização do sistema de saúde, dificuldades para encaminhamento e atendimento), além de elementos sobre a vivência individual daqueles envolvidos no tratamento. Compreender os fatores psicossociais que atuam no episódio de câncer pediátrico promove subsídios para o desenvolvimento e realização de intervenções eficientes, que possam diminuir os custos emocionais e instrumentais característicos desse contexto.

\section{Referências}

Araújo, T. C. C. F. (2006). Câncer infantil: intervenção, formação e pesquisa em Psico-oncologia pediátrica. Psicologia Hospitalar, 4 (1), 1-12.

Araújo, T. C. C. F. \& Guimarães, T. B. (2009). Interações entre voluntários e usuários em onco-hematologia pediátrica: um estudo sobre os "palhaçosdoutores". Estudos e Pesquisas em Psicologia, 9 (3), 632-647.

Avanci, B. S.; Carolindo, F. M.; Góes, F. G. B. \& Netto, N. P. C. (2009). Cuidados paliativos à criança oncológica na situação do viver/morrer: a ótica do cuidar em enfermagem. Escola Anna Nery Revista de Enfermagem, 13 (4), 708-716.

Beck, A. R. M. \& Lopes, M. H. B. M. (2007a). Cuidadores de crianças com câncer: aspectos de vida afetados pela atividade de cuidador. Revista Brasileira de Enfermagem, 60 (6), 670-675.

Beck, A. R. M. \& Lopes, M. H. B. M. (2007b). Tensão devido ao papel de cuidador entre cuidadores de crianças com câncer. Revista Brasileira de Enfermagem, 60 (5), 513-518.

Beltrão, M. R. L. R.; Vasconcelos, M. G. L.; Pontes, C. M. \& Albuquerque, M. C. (2007). Câncer infantil: percepções maternas e estratégias de enfrentamento. Jornal de Pediatria, 83 (6), 562-566.

Bolze, S. D. A. \& Castoldi, L. (2005). O acompanhamento familiar antes e depois da morte da criança: uma proposta de intervenção para o psicólogo hospitalar. Aletheia, 21, 79-91. 
Camacho, M. R. Memórias de um tempo junto a crianças com câncer. Psicologia, Ciência e Profissão, 26 (2), 176-189.

Campos, E. M. P.; Rodrigues, A. L.; Machado, P. \& Alvarez, M. (2007). Intervenção em grupo: experiência com mães de crianças com câncer. Psicologia em Estudo, 12 (3), 635-640.

Capitão, C. G.; Zampronha, M. A. G. (2004). Câncer na adolescência: um estudo com instrumento projetivo. Revista da SBPH, 7 (1), 3-16.

Carlesso, D. R. D. (2008). Desamparo diante da perda do filho. Revista da SBPH, 11 (1), 131-141.

Carvalho, M. M. (2002). Psico-oncologia: história, características e desafios. Psicologia USP, 13 (1), 151-166.

Cavicchioli, A. C.; Menossi, M. J. \& Lima, R. A. G. (2007). Câncer infantil: o itinerário diagnóstico. Revista Latino-americana de Enfermagem, 15 (5), 1025-1032.

Costa, C. L. (2002). Câncer infantil: a realidade da doença na fantasia dos contos de fadas. Acta Oncológica Brasileira, 22 (2), 292-294.

Costa, J. C. \& Lima, R. A. G. (2002). Crianças/adolescentes em quimioterapia ambulatorial: implicações para a enfermagem. Revista Latino-americana de Enfermagem, 10 (3), 321-333.

Costa Junior, A. L. (1999). Psico-oncologia pediátrica: definição e subsídios para atuação profissional. Pediatria Moderna, 35 (6), 442-446.

Costa Junior, A. L. (2001a). Análise de comportamentos de crianças expostas à punção venosa para quimioterapia. Programa de Pós-graduação em Psicologia. Tese de Doutoramento, Universidade de Brasília, Brasília.

Costa Junior, A. L. (2001b). O desenvolvimento da Psico-oncologia: implicações para a pesquisa e intervenção profissional em saúde. Psicologia, Ciência e Profissão, 21 (2), 36-43.

Costa Junior, A. L. \& Kanitz, S. (2000). Avaliação do processo de enfrentamento em crianças com câncer: pesquisa para implantação de programa de intervenção psicológica. Pediatria Moderna, 36 (10), 699-703. 
Costa Junior, A. L., Coutinho, S. M. G. \& Ferreira, R. S. (2006). Recreação planejada em sala de espera de uma unidade pediátrica: efeitos comportamentais. Paideia, 16 (3), 111-118.

Costa Junior, A. L.; Coutinho, S. M. G. \& Kanitz, S. (2000). O enfrentamento do câncer em crianças: a intervenção da Psicologia. Pediatria Moderna, 36 (5), 330-333.

Costa Junior, A. L.; Coutinho, S. M. G.; Ferreira, R. S.; Prado, J. A.; Viana, K. F. \& Meiçó, C. A. (2006). A importância de atividades de recreação em sala de espera de unidade de onco-hematologia pediátrica. Pediatria Moderna, 42 (3), 138-141.

Delella, L. A. \& Araújo, T. C. C. F. (2002). Câncer na infância: uma investigação sobre a avaliação da desordem de estresse pós-traumático parental e a experiência da sobrevivência. Psicologia Argumento, 20 (31), 42-48.

Espíndula, J. A. \& Valle, E. R. M. (2002). Experiência materna diante da iminência de morte do filho com recidiva de câncer. Pediatria Moderna, 38 (5), 188-194.

Ferreira, B. E. S. \& Garcia, A. (2008). Aspectos da amizade de adolescentes portadores de diabetes e câncer. Estudos de Psicologia, 25 (2), 293-301.

Fontenele, M. F. S.; Pinto, V. B.; Andrade, F. J. M.; Dias, A. P.; Moura, R. M. G. \& Pinto, J. M. B. (2000). A biblioterapia no tratamento do câncer infantil. Diálogo Cientifico, 1-24.

Freire, M. C. B.; Petrilli, A. S. \& Sonsogno, M. C. (2007). Humanização em Oncologia Pediátrica: novas perspectivas na assistência ao tratamento do câncer infantil. Pediatria Moderna, 43 (5), 225-236.

Gimenes, M. G. G. (1994). Definição, foco de estudo e intervenção. In: M. M. M. J. Carvalho (org.). Introdução à Psico-oncologia. (pp. 35-56). São Paulo: Psy.

Gottlieb, R.A. \& Pinkel, D. (1991). Manualde Oncologia Pediátrica. (Tradução M. C. A. Souza). Rio de Janeiro: Medsi - Editora Médica e Científica.

Herman, A. R. S. \& Miyazaki, M. C. O. S. (2007). Intervenção psicoeducacional em cuidador de criança com câncer: relato de caso. Arquivos de Ciências da Saúde, 14 (4), 238-244. 
Instituto Nacional do Câncer [Inca]. (2008). Informaçôes sobre o câncer pediátrico. Disponível em <www.inca.gov.br>. (Acesso em 15 fev. 2010).

Jesus, L. K. R. \& Gonçalves, L. L. C. (2006). O cotidiano de adolescentes com leucemia: o significado da quimioterapia. Revista de Enfermagem da UERJ, 14 (4), 545-550.

Kohlsdorf, M. (2008). Análise das estratégias de enfrentamento adotadas por pais de crianças e adolescentes em tratamento de leucemias. Programa de Pósgraduação em Processos de Desenvolvimento Humano e Saúde. Dissertação de Mestrado, Universidade de Brasília, Brasília.

Kohlsdorf, M. \& Costa Junior, A. L. (2008). Estratégias de enfrentamento de pais de crianças em tratamento de câncer. Estudos de Psicologia, 25 (3), 417-429.

Lemos, F. A.; Lima, R. A. G. \& Mello, D. F. (2004). Assistência à criança e ao adolescente com câncer: a fase da quimioterapia intratecal. Revista Latinoamericana de Enfermagem, 12 (3), 485-493.

Malta, J. D. S.; Schall, V. T. \& Modena, C. M. (2008). Câncer pediátrico: o olhar da família/cuidadores. Pediatria Moderna, 44 (3), 114-118.

Malta, J. D. S.; Schall, V. T.; Reis, J. C. \& Modena, C. M. (2009). Quando falar é difícil: a narrativa de crianças com câncer. Pediatria Moderna, 45 (5), 194-198.

Malta, J. D. S.; Schall, V. T. \& Modena, C. M. (2009). O momento do diagnóstico e as dificuldades encontradas pelos oncologistas pediátricos no tratamento do câncer em Belo Horizonte. Revista Brasileira de Cancerologia, 55 (1), 33-39.

Marchesan, E. C.; Bock, A. M. B.; Petrilli, A. S.; Covic, A. N. \& Kanemoto, E. (2009). A não escola: os sentidos atribuídos à escola e ao professor hospitalares por pacientes oncológicos. Psicologia, Ciência e Profissão, 29 (3), 476-493.

Marques, A. P. F. S. (2004). Câncer e estresse: um estudo sobre as crianças em tratamento quimioterápico. Psicologia Hospitalar, 2 (2), 1-12.

Melo, L. L. \& Valle, E. R. M. (2004). A criança com câncer em imin6encia de morte: revisão da literatura. Acta Oncológica Brasileira, 24 (3), 671-675.

Menezes, C. N. B.; Passarelli, P. M.; Drude, F. S.; Santos, M. A. \& Valle, E. R. M. (2007). Câncer infantil: organização familiar e doença. Revista Mal-estar e Subjetividade, 7 (1), 191-210. 
Menossi, M. J.; Lima, R. A. G. \& Corrêa, A. K. (2008). A dor e o desafio da interdisciplinaridade no cuidado à criança. Revista Latino-americana de Enfermagem, 16 (3), 489-494.

Mensório, M. S.; Kohlsdorf, M. \& Costa Junior, A. L. (2009). Cuidadores de crianças e adolescentes com leucemia: análise de estratégias de enfrentamento. Psicologia em Revista, 15 (1), 158-176.

Misko, M. D. \& Bousso, R. S. (2007). Manejando o câncer e suas intercorrências: a família decidindo pela busca ao atendimento de emergências para o filho. Revista Latino-americana de Enfermagem, 15 (1), 48-54.

Morais, L. V. \& Valle, E. R. M. (2001). A terapia ocupacional e a criança hospitalizada. Pediatria Moderna, 37 (5), 177-183.

Moreira, G. M. S. \& Valle, E. R. M. (1999). Estudos bibliográficos sobre publicações brasileiras relacionadas a aspectos psicossociais do câncer infantil no período de 1980 a 1997. Revista Brasileira de Cancerologia, 45 (2), 27-35.

Moreira, P. L. \& Ângelo, M. (2008). Tornar-se mãe de criança com câncer: construindo a parentalidade. Revista Latino-americana de Enfermagem, 16 (3), 355-361.

Motta, A. B. \& Enumo, S. R. F. (2002). Brincar no hospital: câncer infantil e avaliação do enfrentamento da hospitalização. Psicologia, Saúde \& Doenças, I, 3 (1), 23-41.

Motta, A. B. \& Enumo, S. R. F. (2004a). Brincar no hospital: estratégia de enfrentamento da hospitalização infantil. Psicologia em Estudo, 9 (1), 19-28.

Motta, A. B. \& Enumo, S. R. F. (2004b). Câncer infantil: uma proposta de avaliação das estratégias de enfrentamento da hospitalização. Estudos de Psicologia, 21 (3), 193-202.

Mussa, C. \& Malerbi, F. E. K. (2008). O impacto da atividade lúdica sobre o bem-estar de crianças hospitalizadas. Psicologia: Teoria e Prática, 10 (2), 83-93.

Nascimento, L. C.; Rocha, S. M. M.; Hayes, V. H. \& Lima, R. A. G. (2005). Crianças com câncer e suas famílias. Revista da Escola de Enfermagem da USP, 39 (4), 469-474.

Ortiz, M. C. A. \& Lima, R. A. G. (2007). Experiência de familiares de crianças e adolescentes, após o término do tratamento contra câncer: subsídios para o cuidado de enfermagem. Revista Latino-americana de Enfermagem, 15 (3), 411-417. 
Paro, D.; Paro, J. \& Ferreira, D. L. M. (2005). O enfermeiro e o cuidar em oncologia pediátrica. Arquivos de Ciências da Saúde, 12 (3), 151-157.

Patenaude, A. F. \& Kupst, M. J. (2005). Psychosocial functioning in pediatric cancer. Journal of Pediatric Psychology, 30 (1), 9-27.

Pedro, E. N. R. \& Funghetto, S. S. (2005). Concepções de cuidado para os cuidadores: um estudo com a criança hospitalizada com câncer. Revista Gaúcha de Enfermagem, 26 (2), 210-219.

Pedro, I. C. S.; Galvão, C. M.; Rocha, S. M. M. \& Nascimento, L. C. (2008). Apoio social e famílias de crianças com câncer: revisão integrativa. Revista Latino-americana de Enfermagem, 16 (3), 477-483.

Pedrosa, A. M.; Monteiro, H.; Lins, K.; Pedrosa, F. \& Melo, C. (2007). Diversão em movimento: um projeto lúdico para crianças hospitalizadas no Serviço de Oncologia Pediátrica do Instituto Materno Infantil Prof. Fernando Figueira, IMIP. Revista Brasileira de Saúde Materno-Infantil, 7 (1), 99-106.

Pedrosa, C. M. \& Valle, E. R. M. (2000). Ser irmão de criança com câncer: estudo compreensivo. Pediatria, 22 (2), 185-194.

Ramalho, M. A. N. \& Nogueira-Martins, M. C. F. (2007). Vivências de profissionais de saúde da área de Oncologia Pediátrica. Psicologia em Estudo, 12 (1), 123-132.

Remedi, P. P.; Mello, D. F.; Menossi, M. J. \& Lima, R. A. G. (2009). Cuidados paliativos para adolescentes com câncer: uma revisão da literatura. Revista Brasileira de Enfermagem, 62 (1), 107-112.

Ribeiro, I. B. \& Rodrigues, B. M. R. D. (2005). Cuidando de adolescentes com câncer: contribuiçôes para o cuidar em enfermagem. Revista de Enfermagem da UERJ, 13, 340-346.

Rodrigues, C. D. S.; Culau, J. M. C. \& Nunes, D. M. (2007). Aprendendo a cuidar: vivências de estudantes de enfermagem com crianças portadoras de câncer. Revista Gaúcha de Enfermagem, 28 (2), 274-282.

Santos, L. M. P. \& Gonçalves, L. L. C. (2008). Crianças com câncer: desvelando o significado do adoecimento atribuído por suas mães. Revista de Enfermagem da UERJ, 16 (2), 224-229. 
Silva, A. M.; Gallego, E. T. \& Teixeira, M. C. T. V. (2006). Habilidades intelectuais de crianças com câncer e crianças não portadoras da doença. Avaliação Psicológica, 5 (1), 33-41.

Silva, F. A. C.; Andrade, P. R.; Barbosa, T. R.; Hoffmann, M. V. \& Macedo, C. R. (2009). Representação do processo de adoecimento de crianças e adolescentes oncológicos junto aos familiares. Escola Anna Nery Revista de Enfermagem, 13 (2), 334-341.

Silva, G. M. \& Valle, E. R. M. (2005). A vivência da reinserção escolar de crianças com câncer: estratégias de atuação do psicólogo. Revista da Associação Brasileira de Psicopedagogia, 22 (67), 2-13.

Silva, G. M.; Teles, S. S. \& Valle, E. R. M. (2005). Estudo sobre as publicações brasileiras relacionadas a aspectos psicossociais do câncer infantil: período de 1998 a 2004. Revista Brasileira de Cancerologia, 51 (3), 253-261.

Silveira, C. S. \& Lima, R. A. G. (2001). Levantamento sobre casas de apoio à criança com câncer. Pediatria Moderna, 37 (1/2), 18-23.

Souza, A. I. J. \& Erdmann, A. L. (2003). A criança com diagnóstico de câncer: revisitando o caminho das políticas de atendimento. Revista Gaúcha de Enfermagem, 24 (1), 23-33.

Steffen, B. C. \& Castoldi, L. (2006). Sobrevivendo à tempestade: a influência do tratamento oncológico de um filho na dinâmica conjugal. Psicologia, Ciência e Profissão, 26 (3), 406-425.

Teles, S. S. \& Valle, E. R. M. (2009). Adulto sobrevivente de câncer infantil: uma revisão bibliográfica. Psicologia em Estudo, 14 (2), 355-363.

Vasconcelos, R. F.; Albuquerque, V. B. \& Costa, M. L. G. (2006). Reflexões da clínica terapêutica ocupacional junto à criança com câncer na vigência da quimioterapia. Revista Brasileira de Cancerologia, 52 (2), 129-137.

Veras, L. \& Moreira, V. (2009). A compreensão do mundo vivido da criança sertaneja com câncer: contribuições do livro "Dudu vai ao hospital". Revista da SBPH, 12 (1), 3-16.

Wegner, W. \& Pedro, E. N. R. (2009). Concepções de saúde sob a ótica de mulheres cuidadoras leigas, acompanhantes de crianças hospitalizadas. Revista Latino-americana de Enfermagem, 17 (1), 88-93. 